Introduction Occupational asthma (OA), obstructive chronic bronchopneumonia (OCBP) and pneumoconiosis are occupational respiratory diseases having important impact on morbidity and mortality. Some population studies show that $15 \%$ of the cases of asthma with the adults can be attributed to work environment. The aims of this study is to evaluate the respiratory function especially the occupational asthma of workers in a plastic bag manufacturing factory in Togo.

Material and methods By a cross sectional study, we included 182 workers in the sample. They filled a questionnaire and the workers with asthma complains was submitted to spirometry test.

Results The mean age of subjects was $37,13 \pm 5,71$ years with a male predominance $(99,5 \%)$. The mean of the work experience was $7,66 \pm 2,88$ years. $1,1 \%$ of workers were smokers. Rhinitis and conjunctivitis were reported with $79,7 \%$. The frequency of occupational rhinitis was $62,6 \%$. Subjects that presented asthma complains were $34,1 \%$. In $25,8 \%$ of cases asthma complains are associated to the work. The abnormal pulmonary function was overlooked by reversible smalls airways pattern $(6,6 \%)$ and reversible obstructive pattern $(4,9 \%)$. Spirometry was compatible with asthma in $11,5 \%$ of cases. The prevalence of occupational asthma was 7,7\%. Occupational asthma only concerns the posts of Thermo-welding and Extrusion. All the workers in these posts did not use respiratory protection.

Conclusion Occupational asthma is a reality in a plastic bag manufacturing factory in Togo. This fact needs preventives and correctives actions to avoid that disease.

\section{A FREELY-AVAILABLE TUBERCULOSIS SCREENING TOOL FOR ADULT WORKERS EXPOSED TO RESPIRABLE CRYSTALLINE SILICA}

${ }^{1}$ NK Reul*, ${ }^{2}$ B Braid, ${ }^{3} \mathrm{Z}$ Gray. ${ }^{1}$ Associate Medical Director for Occupational Disease, Washington State Department of Labour and Industries, Olympia, Washington, USA; ${ }^{2}$ Clinical Nurse Specialist, Washington State Department of Labour and Industries, Olympia, Washington, USA; ${ }^{3}$ Epidemiologist, Washington State Department of Labour and Industries, Olympia, Washington, USA

\subsection{6/oemed-2018-ICOHabstracts. 1306}

Introduction Exposure to respirable crystalline silica is known to increase the risk of progression to active tuberculosis (TB) disease in those who have latent TB infection. Both latent TB infection and active $\mathrm{TB}$ disease are relevant components of screening examinations in silica-exposed workers.

Methods Health care providers screening silica-exposed workers for TB must generally:

- identify for further evaluation and treatment any employees with suspected active TB disease

- adhere to safety and health regulatory requirements, when applicable

- even when regulations do not require screening, exercise sound screening practices in workers who stand to benefit from medical evaluations

- be familiar with current peer-reviewed publications; specialty society recommendations; national and state public health policy approaches

- coordinate with local approaches to TB screening
- incorporate both occupational and non-occupational health indications into screening decisions.

Using a publicly-funded, transparent, and democratic process, the Department of Labour and Industries has developed a TB risk assessment and testing algorithm for adult workers exposed to respirable crystalline silica.

The screening tool integrates these varied needs and resources into a cohesive document for use at the point of health care delivery.

Result The tool is a clinical adjunct that helps health care providers make two key decisions when evaluating patients with exposure to respirable crystalline silica:

1. Which workers should undergo a comprehensive evaluation for active tuberculosis disease?

2. Which workers should receive testing for latent TB infection?

Discussion Widespread availability of the risk assessment and testing algorithm is assured through direct inclusion in Washington State regulatory documents. The tool integrates medical content and public policy for clinicians performing the TB screening in silica-exposed workers through a single, accessible form. As a freely-available clinical aid, it is available to clinicians performing TB screening in silica-exposed workers throughout the United States, and worldwide.

\section{BREATH TEST FOR PNEUMOCONIOSIS USING AN ELECTRONIC NOSE: A CASE-CONTROL STUDY}

${ }^{1}$ Hsin-Yi Peng ${ }^{*}$, ${ }^{1}$ Che-Jui Chang, ${ }^{1,2,3,4}$ Pau-Chung Chen, ${ }^{1,2,3} \mathrm{Hsiao}-Y u$ Yang. ${ }^{1}$ Institute of Occupational Medicine and Industrial Hygiene, National Taiwan University College of Public Health, Taipei, Taiwan; ${ }^{2}$ Department of Public Health, National Taiwan University College of Public Health, Taipei, Taiwan; ${ }^{3}$ Department of Environmental and Occupational Medicine, National Taiwan University Hospital, Taipei, Taiwan; ${ }^{4}$ Department of Environmental and Occupational Medicine, National Taiwan University College of Medicine, Taipei, Taiwan

\subsection{6/oemed-2018-ICOHabstracts. 1307}

Introduction Current medical surveillance program has flaw that may result in poor detection of early pneumoconiosis around the world. Breath analyses have attracted substantial attention in the screening of occupational environmental lung disease. Pneumoconiosis could generate specific volatile organic compounds (VOCs) that may constitute a specific breath print for diagnosis. The objective of this study was to develop a breath test for pneumoconiosis by analysing VOCs using senor array technique.

Methods We conducted a case-control study and enrolled study subjects from stone workers in Hualien between October 2016 and November 2016. One litter of breath air was collected after five minutes of tidal breathing through a nonrebreathing valve with inspiratory VOC-filter, and storage by a Tedlar bag. The air was analysed by a 32 nanocomposite sensor array electronic nose within $30 \mathrm{~min}$. Using the ILO/ICRP profusion category $\geq 1 / 1$ in chest $\mathrm{X}$-ray as the reference standard, we assessed the diagnostic accuracy of the breath test. Data were randomly split into $80 \%$ for model building and $20 \%$ for validation.

Result After excluding three subjects with poorly controlled diabetes mellitus with fasting serum glucose level $>200 \mathrm{mg} / \mathrm{dl}$ and one subjects of asthma under medication, a total of 98 subjected were used in final analysis that included 34 cases of pneumoconiosis and 64 healthy controls. By linear 
discriminant analysis, the sensitivity was $88.0 \%$, specificity was $67.9 \%$, accuracy was $80.8 \%$, and ROC-AUC was $0.91(95 \%$ CI: 0.85 to 0.97$)$ in the training set. In the validation set, the sensitivity was $66.7 \%$, specificity was $71.4 \%$, accuracy was $70.0 \%$, and ROC-AUC was 0.86 (95\% CI: 0.69 to 1.00 ).

Discussion Breath test may have potential in screening for pneumoconiosis. A multi-centre study is warranted to establish a reliable model and the procedures must be standardised to prevent confounding factors before clinical application.

\section{ASBESTOSIS AND GENE-ENVIRONMENT INTERACTIONS}

${ }^{1} \mathrm{~A}$ Franko, ${ }^{2} \mathrm{~V}$ Dolzan, ${ }^{1} \mathrm{M}$ Dodic-Fikfak. ${ }^{1}$ Clinical Institute of Occupational Medicine, University Medical Centre, Ljubljana, Slovenia; ${ }^{2}$ Pharmacogenetics Laboratory, Institute of Biochemistry, Faculty of Medicine, University of Ljubljana, Slovenia

\subsection{6/oemed-2018-ICOHabstracts. 1308}

Introduction Although the causal relationship between asbestosis and asbestos exposure has been well proved, little is known about the genetic factors that may influence the development of this disease. This study investigated the influence of gene-gene and geneenvironment interactions on the risk of developing asbestosis.

Methods The nested case-control study included 262 cases with asbestosis and 265 controls with no asbestos-related disease studied for MnSOD, ECSOD, CAT, GSTT1, GSTM1, GSTP1, and iNOS polymorphisms. Data on cumulative asbestos exposure and smoking were available for all subjects. PCR based methods were used for genotyping. Logistic regression analysis was used to asses asbestosis risk.

Result The OR of asbestosis was 3.21 (95\% CI: 2.43 to 4.23 ) for cumulative asbestos exposure; 0.98 (95\% CI: 0.69 to 1.39) for smoking; 1.50 (95\% CI: 1.01 to 2.24) for $M n S O D$ -9Ala/Ala versus $\mathrm{Ala} / \mathrm{Val}$ and $\mathrm{Val} / \mathrm{Val} ; 1.63$ (95\% CI: 0.62 to 4.27) for ECSOD 213Arg/Gly versus Arg/Arg; 1.36 (95\% CI: 0.70 to 2.62$)$ for CAT -262TT versus CT and CC; 1.20 (95\% CI: 0.85 to 1.69 ) for $i$ NOS LL versus SL and SS; 1.01 (95\% CI: 0.71 to 1.43 ) for GSTM1-null; 0.61 (95\% CI: 0.40 to 0.94 ) for GSTT1-null; 1.52 (95\% CI: 1.08 to 2.15 ) for GSTP1 105Ile/Ile versus 105Ile/Val and 105 Val/Val; and 0.97 (95\% CI: 0.64 to 1.48 ) for GSTP1 114Ala/Ala versus 114Ala/ $\mathrm{Val}$ and $114 \mathrm{Val} / \mathrm{Val}$. The associations between MnSOD Ala9 Val polymorphism and asbestosis, and between iNOS genotypes and asbestosis $(\mathrm{p}=0.038)$ were modified by CAT$262 \mathrm{C}>\mathrm{T}$ polymorphism $(\mathrm{p}=0.031)$. A strong interaction was found between GSTM1-null polymorphism and smoking $(\mathrm{p}=0.007), \quad$ iNOS $(\text { CCTTT })_{\mathrm{n}}$ polymorphism and smoking $(\mathrm{p}=0.054)$ as well as between $i$ NOS $(\text { CCTTT })_{n}$ polymorphism and cumulative asbestos exposure $(\mathrm{p}=0.037)$.

Discussion The findings suggest that in addition to asbestos exposure the interactions between different genotypes, genotypes and smoking, and between genotypes and asbestos exposure have an important influence on developing asbestosis and should be considered seriously in future research on occupational/environmental asbestos-related diseases.

\section{2}

SPIROMETRY LONGITUDINAL DATA ANALYSIS SOFTWARE (SPIROLA) FOR RESPIRATORY DISEASE PREVENTION AMONG SHIPYARD WELDERS IN SOUTH KOREA

${ }^{1}$ Se Yeong Kim*, ${ }^{2}$ Dong Mug Kang, ${ }^{3}$ Jung II Kim. 'Busan Workers' Health Centre and Pusan National University, Pusan, Korea; ${ }^{2}$ Occupational and Environmental Medicine, Pusan National University Yangsan Hospital, Pusan, Korea; ${ }^{3}$ Occupational and Environmental Medicine, Dong- A University Hospital, Pusan, Korea

\subsection{6/oemed-2018-ICOHabstracts. 1309}

Introduction Workplace health monitoring using periodic spirometry has been recommended as a tool for prevention of respiratory disease. The Spirometry Longitudinal Data Analysis (SPIROLA) software is an integrated visual and quantitative tool to aid in monitoring lung function in individuals participating in spirometry-based health monitoring programs developed by Centres for Disease Control and Prevention (CDC).

Methods We analysed the annual lung function decline in 385 male workers of the Korean Shipyard Welder Cohorts by SPIROLA. To evaluate the annual decline of spirometry data, we analysed the longitudinal spirometry data collected from 2010 through the year 2015 in workers who had at least four follow-up tests. We estimated each person's rate of $\mathrm{FEV}_{1}$ and FVC decline and group's rate of $\mathrm{FEV}_{1}$ and FVC decline.

Result The results on 385 workers with 4 or more years of follow-up showed that the mean rate of $\mathrm{FEV}_{1}$ decline was higher than that for the Korean population. And decline of lung function in welders suggest a greater effect in smoking status.

Discussion This study indicates the need for respiratory disease prevention and intervention in this shipyard welders. The computerised annual lung function decline program is very useful of workers' respiratory health prevention. So We need to develop a program suitable for workplace environment in Korean.

\section{WORK-RELATED ASTHMA AMONGST ASTHMATIC PATIENTS IN THE EASY ASTHMA CLINICS IN KHON KAEN, THAILAND}

${ }^{1} \mathrm{~N}$ Chaiear* ${ }^{*}{ }^{1} \mathrm{~W}$ Jongkumchok, ${ }^{2} \mathrm{~W}$ Boonsawat, ${ }^{3} \mathrm{~S}$ Chaisuksant. ${ }^{1}$ Division of Occupational Medicine, Department of Community Medicine, Faculty of Medicine, Khon Kaen University Khon Kaen, Thailand; ' 2 Department of Medicine, Faculty of Medicine, Khon Kaen University, Khon Kaen, Thailand; ${ }^{3}$ Department of Medicine, Khon Kaen Hospital, Khon Kaen, Thailand

\subsection{6/oemed-2018-ICOHabstracts. 1310}

Introduction Work-related asthma (WRA) amongst asthmatic patients in worldwide country are about 15\%-25\%. In Thailand, there are at least 3 million asthmatics patients but there have never been a figure of WRA presented. This is the first study in Thailand where a proportion of WRA amongst asthmatic patients was figured.

Methods A descriptive study was performed. The samples of 523 asthmatic patients in the Easy Asthma Clinics were 